\title{
Glucose and lactose as cryoprotectants for fungal strains immobilised in sodium alginate: an emphasis on the conservation of the zygomycetes Rhizopus and Mucor
}

\author{
Marcos F. G. Rocha, ${ }^{1,2,3}$ Daniel T. Lima, ${ }^{1,2}$ Raimunda S. N. Brilhante, ${ }^{1,2,4}$ Rossana A. Cordeiro, ${ }^{1,2,4}$ \\ André J. Monteiro, ${ }^{5}$ Carlos E. C. Teixeira, ${ }^{1,2}$ Joyce F. Ribeiro, ${ }^{1,2}$ Débora S. C. M. Castelo-Branco ${ }^{1,2}$ and \\ José J. C. Sidrim ${ }^{1,2,3}$ \\ ${ }^{1}$ Specialized Medical Mycology Center, Federal University of Ceará, Fortaleza, Brazil, ${ }^{2}$ Postgraduate Program in Medical Microbiology, Federal University of \\ Ceará, Fortaleza, Brazil, ${ }^{3}$ Postgraduate Program in Veterinary Science, State University of Ceará, Fortaleza, Brazil, ${ }^{4}$ Postgraduate Program in Medical Sciences, \\ Federal University of Ceará, Fortaleza, Brazil and ${ }^{5}$ Department of Statistics and Applied Mathematics, Federal University of Ceará, Fortaleza, Brazil
}

\section{Summary}

\begin{abstract}
This research aimed at investigating the cryoprotectant action of glucose and lactose on strains of Malassezia spp. and zygomycetes immobilised in sodium alginate. Twelve strains of Malassezia spp. (nine M. furfur, two M. globosa and one M. sympodialis) and 12 zygomycetes (five Rhizopus oryzae and seven Mucor hiemales) were immobilised in sodium alginate, within plastic beads, maintained in appropriate media containing glucose and lactose at concentrations of $9 \%$ and $23 \%$ and preserved at temperatures of -20 and $-80^{\circ} \mathrm{C}$. Strain viability was evaluated from 15 to 270 days of storage, through the observation of macro-micromorphologic characteristics. The Malassezia spp. strains were only viable until 90 days of storage, whereas for zygomycetes, viable strains were observed until after 270 days of storage at $-80{ }^{\circ} \mathrm{C}$, in the media containing $23 \%$ glucose or lactose. The use of $23 \%$ glucose or lactose at $-80{ }^{\circ} \mathrm{C}$ in a sodium alginate cell immobilisation system is efficient for cryopreserving zygomycetes. This research creates perspectives for the use of glucose and lactose in sodium alginate cell immobilisation systems for the preservation of fungi with low viability.
\end{abstract}

Key words: Zygomycetes, Malassezia, storage, sodium alginate, glucose and lactose.

\section{Introduction}

The genus Malassezia comprises lipophilic yeast that belong to the normal cutaneous microbiota of humans and other warm blooded animals, but these yeast are also associated with a variety of clinical manifestations, such as pityriasis versicolor, seborrhoeic dermatitis and, in rare situations, fungaemia. ${ }^{1,2}$ Despite presenting a wide variety of hosts, this yeast genus does not play an important role in agriculture. The genera Mucor and

Correspondence: R. S. N. Brilhante, Rua Barão de Canindé, 210, Montese, CEP: 60.425-540 Fortaleza, Brazil.

Tel.: +55 (85) 3366 8319. Fax: +55 (85) 32951736.

E-mail: brilhante@ufc.br

Submitted for publication 6 May 2012

Revised 9 September 2012

Accepted for publication 8 November 2012
Rhizopus belong to the order Mucorales and the phylum Zygomycota and are included in the group of the Zygomycetes. In humans and other animals, these fungi can cause zygomycosis, which is most commonly characterised by rhinocerebral and/or invasive angiotropic infections. ${ }^{3,4}$ In agriculture, R. stolonifer is the agent of rhizopus rot disease that affects several vegetables and fruits. ${ }^{5}$

As observed in our laboratory and by other authors, there are difficulties in preserving zygomycetes and Malassezia spp. ${ }^{1,6}$ Zygomycetes form a fungal group whose hyphae are wide $(6-20 \mu \mathrm{m})$, thin-walled, relatively large and rarely septate that present low cellular protein content. As a consequence, they frequently collapse, exhibiting an appearance of a braided tape, as observed under microscopy. ${ }^{3,4}$ These characteristics make them more vulnerable to mechanical damages, including those caused by ice crystals. Concerning the 
genus Malassezia spp., their low in vitro viability represents the main obstacle for retrospective and prospective studies, as reported by several authors. ${ }^{1,6,7}$ Thus, the development of an efficient methodology for the preservation of zygomycetes and Malassezia spp. is of great importance for the development of prospective studies with these microorganisms.

It has been reported that the occurrence of mutations and the loss of fungal viability decreases when cryopreservation is used. This happens because cell metabolic activity declines and life expectancy increases as the temperature reaches $0{ }^{\circ} \mathrm{C} .{ }^{8}$ Among the techniques of preserving microorganisms, vitrification is a feasible alternative to the Castelani method. In vitrification, the water shifts from the liquid to an amorphous and metastable solid state. ${ }^{9}$ This situation promotes protection during freezing because the formation of a vitreous state induces sufficient viscosity within and around the cell, stabilising molecular mobility. It has also been reported that carbohydrates, such as glucose, lactose and trehalose, are cryoprotectants that promote vitrification, hence preventing cellular lesions that occur after freezing of cell fluids, through interception of salts, because of the high viscosity or the formation of a vitreous phase. ${ }^{10}$ It is important to highlight that immobilisation and cryopreservation have been successfully used in cryobanks for the conservation of the phytodiversity. ${ }^{11}$ Several authors have reported that alginate is an inert and low-cost chemical support capable of immobilising microbial and plant cells. ${ }^{11-15}$ Thus, the objective of this study was to investigate the cryoprotectant effect of glucose and lactose on strains of Malassezia spp. and zygomycetes immobilised in sodium alginate.

\section{Materials and methods}

Twelve strains of Malassezia (nine M. furfur, two $M$. globosa and one M. sympodialis) and 12 strains of zygomycetes (five Rhizopus oryzae and seven Mucor hiemales), from the culture collection of the Specialized Medical Mycology Center of the Federal University of Ceará, Fortaleza, Ceará, Brazil, were used (Table 1).

The immobilisation of fungal cells was performed through the process of ionic gelation. ${ }^{12} \mathrm{~A}$ calcium chloride solution at $0.25 \mathrm{~mol} \mathrm{l}^{-1}$ was previously frozen (Fig. 1a), within a screw-cap glass tube (16 mm wide $\times 150 \mathrm{~mm}$ length), to form a sustenance surface for the addition of sterile plastic beads $(0.7-\mathrm{cm}$ diameter with a $0.4-\mathrm{cm}$ diameter central orifice) (Fig 1b).

Malassezia spp. strains were cultured on Dixon agar and incubated at $32{ }^{\circ} \mathrm{C}$, and were observed daily for up
Table 1 Collection numbers of the tested strains.

\begin{tabular}{ll}
\hline Collection numbers & Strains \\
\hline Malassezia & \\
CEMM 03-6-065 & M. furfur \\
CEMM 03-6-066 & M. furfur \\
CEMM 03-6-067 & M. sympodialis \\
CEMM 03-6-068 & M. furfur \\
CEMM 03-6-069 & M. furfur \\
CEMM 03-6-070 & M. furfur \\
CEMM 03-6-071 & M. furfur \\
CEMM 03-6-072 & M. furfur \\
CEMM 03-6-073 & M. globosa. \\
CEMM 03-6-074 & M. furfur \\
CEMM 03-6-075 & M. globosa \\
CEMM 03-6-076 & M. furfur \\
ZygOMycetes & \\
CEMM 03-1-030 & Rhizopus orizae \\
CEMM 03-1-067 & Mucor hiemalis \\
CEMM 03-2-006 & R. orizae \\
CEMM 03-4-013 & R. orizae \\
CEMM 03-4-027 & R. orizae \\
CEMM 03-4-086 & M. hiemalis \\
CEMM 03-4-087 & M. hiemalis \\
CEMM 03-4-088 & M. hiemalis \\
CEMM 03-4-089 & M. hiemalis \\
CEMM 03-5-016 & M. hiemalis \\
CEMM 03-5-025 & R. orizae \\
CEMM 05-2-044 & M. hiemalis \\
\hline
\end{tabular}

to 15 days. Afterwards, the cultures were scraped and transferred to a medium formed of $1.5 \%(\mathrm{w} / \mathrm{v})$ sodium alginate, 3\% (w/v) Dixon broth, 9\% or $23 \%(\mathrm{w} / \mathrm{v})$ glucose or $9 \%$ or $23 \%(\mathrm{w} / \mathrm{v})$ lactose and homogenised with a vortex for $30 \mathrm{~s}$. The zygomycete strains were cultured on potato dextrose agar and maintained at $25{ }^{\circ} \mathrm{C}$. They were daily observed for up to 7 days to evaluate culture growth. Then, the cultures obtained were scraped from the agar and transferred to the immobilisation-cryopreservation medium, which contained $1.5 \%$ sodium alginate, 3\% (w/v) Sabouraud broth, $9 \%$ or $23 \%$ glucose and $9 \%$ or $23 \%$ lactose. It is important to emphasise that glucose and lactose were the only cryoprotectants used and the concentrations were chosen based on previous studies. ${ }^{16,17}$ Before and after storage, the strains were evaluated to assure their viability and the maintenance of phenotypical features. Malassezia strains were submitted to metabolic tests, such as assimilation of Tween and Chremophor, urease, catalase and $\beta$-glucosidase production, whereas zygomycete strains were submitted to micromorphological analysis. $^{18-20}$

The immobilisation of the fungal cells was carried out by the ionic gelation process ${ }^{12}$ with modifications involving prior freezing of the calcium chloride and 
Figure 1 (a) Previously frozen calcium chloride; (b) Plastic bead; (c) As the calcium chloride solution thaws and reacts with the mixture of fungal cells with sodium alginate, fungal cell imprisonment occurs within the plastic beads; (d) Storage within cryotubes.

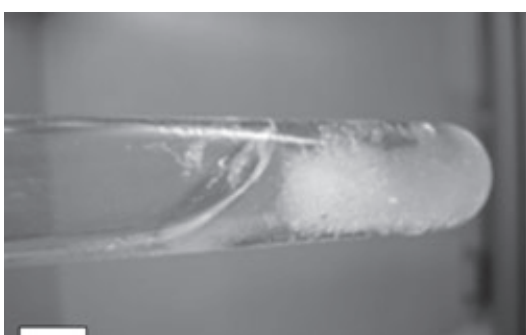

(a)

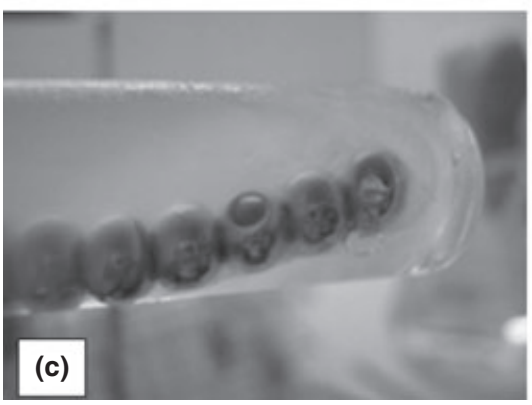

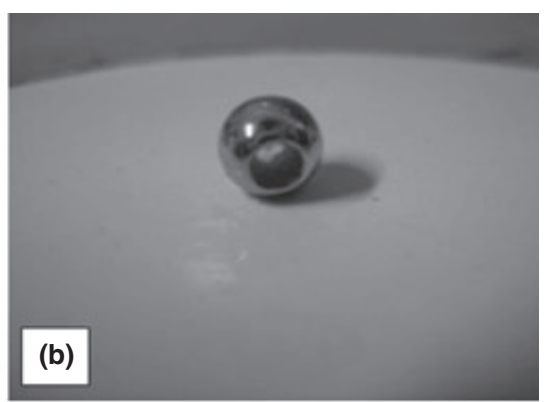

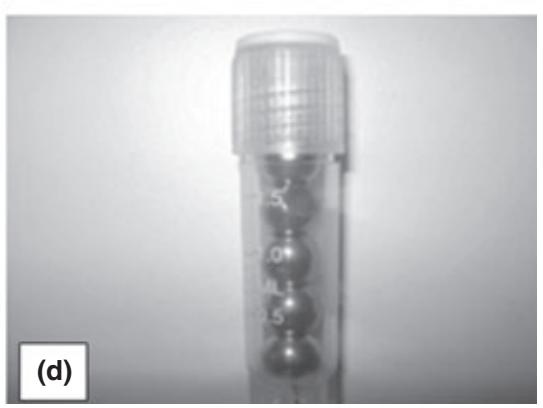

addition of plastic beads for preservation of the fungal structure trapped inside. In brief, the plastic beads were added to the fungal structures homogenised in the immobilisation-cryopreservation solutions and were then placed under a block of frozen calcium chloride (Fig 1c). As the calcium chloride solution thawed, it reacted with the sodium alginate and promoted the gelation and the imprisonment of the fungal structures within the plastic beads. Finally, each culture was fractioned and kept in 1.5-ml, five cryogenic tubes each containing 5 beads (Fig 1d), which were directly placed in the freezer at -20 and $-80{ }^{\circ} \mathrm{C}$. The cultures were evaluated for their viability at 15, 45, 90, 180 and 270 days of storage. Each strain was stored in 5 different cryogenic tubes and, at every evaluation day, beads were removed from only one tube. At the next evaluation day, the beads were removed from a different tube, therefore, each cryogenic tube was evaluated only once. One of the beads was removed under aseptic conditions with a sterile needle, as soon as the cryogenic tube was taken out of the freezer. Then, the bead was inoculated onto either Dixon agar or potato dextrose agar and incubated for at least 15 days, at $25^{\circ} \mathrm{C}{ }^{21}$ To compare the storage strategies, the Fisher exact test was used, at a significance level of $5 \%$.

\section{Results}

Overall, Malassezia spp. presented low resistance to cryopreservation, independently of the medium, the sugar concentration or the temperature used, and they rapidly became unviable. Approximately 94\% of the strains kept in the medium with glucose and 100\% of the strains in the medium containing lactose were still viable at day 15 of storage, but these rates declined to $65 \%$ and $63 \%$, respectively, at day 45 , and to $13 \%$ and $6 \%$ at day 90 of storage. Statistically significant differences between viability percentages were not observed because of the reduced number of tested strains (Table 2).

The zygomycetes presented higher viability when stored at $-80{ }^{\circ} \mathrm{C}$ than at $-20{ }^{\circ} \mathrm{C}$, and no statistic differences were observed between the carbohydrates used and the concentrations tested at the same storage temperature. The number of viable strains kept at $-80{ }^{\circ} \mathrm{C}$ in $23 \%$ glucose $(P=0.01337)$ and $23 \%$ lactose $(P=0.0046)$, at day 270 of storage, was statistically higher than in the other treatments. Due to the small number of strains, no other statistical differences were observed among treatments (Table 2). Viable Malassezia spp. and zygomycetes showed preserved macro-micromorphologic features after storage, as shown in Fig 2.

\section{Discussion}

Despite the difficulty in maintaining zygomycetes in fungal collections, a survival rate of $50 \%$ was observed in this work for strains of Rhizopus sp. and Mucor sp. maintained at $-80{ }^{\circ} \mathrm{C}$ for 270 days, in media containing 23\% glucose or lactose. On the other hand, by applying the same methodology, viable strains of Malassezia were only recovered until 90 days of storage, and at low recovery rates. This evidence once again 
M. F. G. Rocha et al.

Table 2 Course of the cryoprotectant action of glucose and lactose on fungal strains immobilised in sodium alginate and maintained at different storage temperatures

\begin{tabular}{|c|c|c|c|c|c|c|c|c|c|}
\hline & \multirow{2}{*}{$\begin{array}{l}\text { Storage } \\
\text { time (days) }\end{array}$} & \multicolumn{2}{|c|}{$-20^{\circ} \mathrm{C}$ Glucose } & \multicolumn{2}{|c|}{$-80^{\circ} \mathrm{C}$ Glucose } & \multicolumn{2}{|c|}{$-20^{\circ} \mathrm{C}$ Lactose } & \multicolumn{2}{|c|}{$-80^{\circ} \mathrm{C}$ Lactose } \\
\hline & & $9 \%$ & $23 \%$ & $9 \%$ & $23 \%$ & $9 \%$ & $23 \%$ & $9 \%$ & $23 \%$ \\
\hline \multirow[t]{5}{*}{ Malassezia spp. $(n=12)$} & 15 & 10 & 11 & 12 & 12 & 12 & 12 & 12 & 12 \\
\hline & 45 & 08 & 07 & 08 & 08 & 07 & 07 & 08 & 08 \\
\hline & 90 & 00 & 01 & 02 & 03 & 00 & 00 & 01 & 02 \\
\hline & 180 & 00 & 00 & 00 & 00 & 00 & 00 & 00 & 00 \\
\hline & 270 & 00 & 00 & 00 & 00 & 00 & 00 & 00 & 00 \\
\hline \multirow[t]{5}{*}{ Zygomycetes $(n=12)$} & 15 & 08 & 09 & 11 & 11 & 09 & 08 & 10 & 11 \\
\hline & 45 & 04 & 06 & 06 & 09 & 08 & 06 & 09 & 10 \\
\hline & 90 & 04 & 05 & 06 & 08 & 06 & 03 & 05 & 08 \\
\hline & 180 & 01 & 01 & 04 & 08 & 03 & 01 & 05 & 08 \\
\hline & 270 & 00 & 00 & 04 & 06 & 01 & 00 & 05 & 07 \\
\hline
\end{tabular}
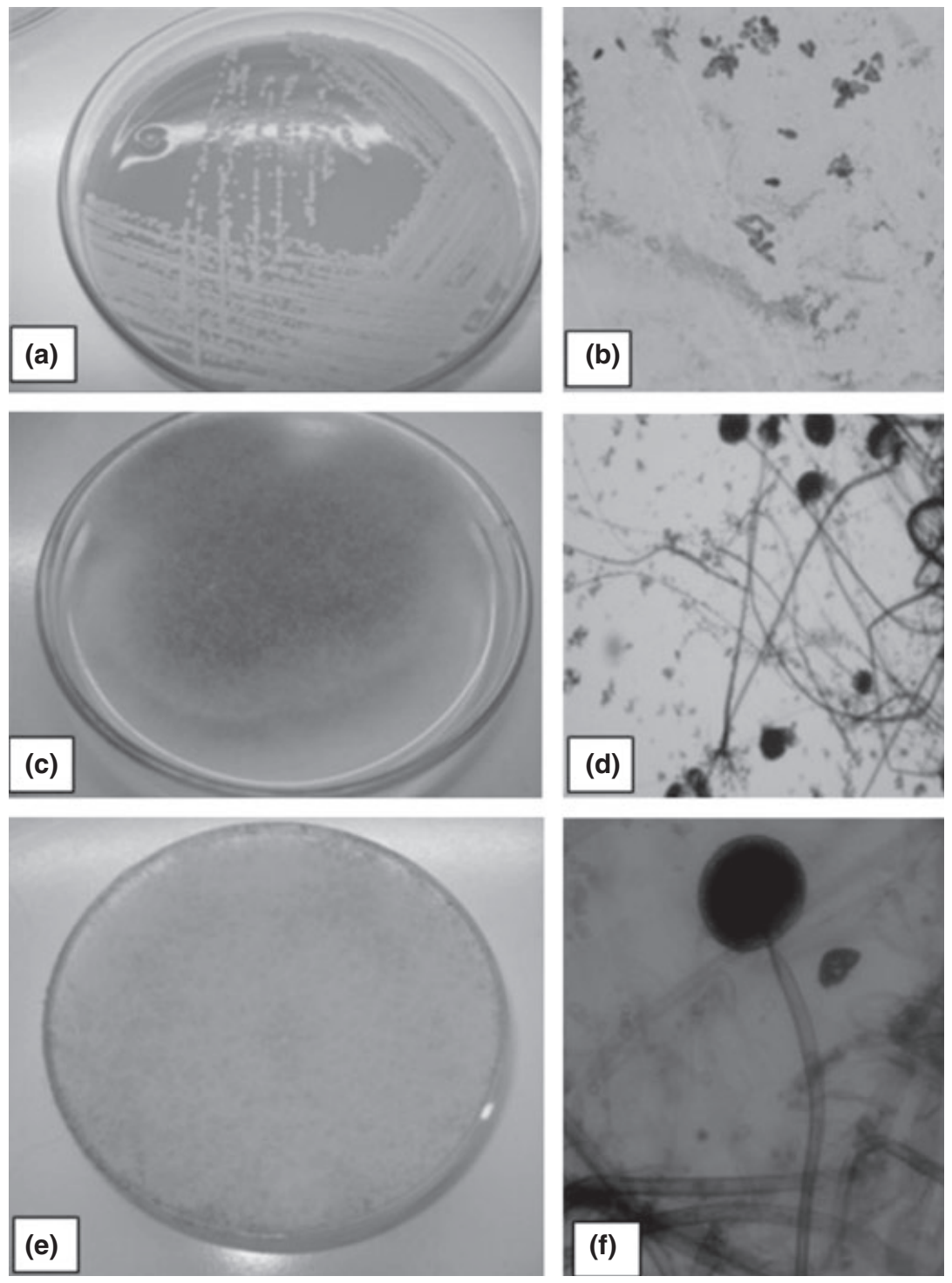

Figure 2 Macromorphology (a) and micromorphology (b) of Malassezia sp., after 45 days of storage at $-80^{\circ} \mathrm{C}$, using $23 \%$ glucose. Macromorphology (c) and micromorphology (d) of Rhizopus sp. after 180 days of storage at $-80{ }^{\circ} \mathrm{C}$, using $23 \%$ glucose. Macromorphology (e) and micromorphology (f) of Mucor sp. after 270 days of storage at $-80{ }^{\circ} \mathrm{C}$, using $23 \%$ glucose. 
confirms that there is no standard protocol for the conservation of fungi in culture collections, as these microorganisms have widely varied physiologic and biochemical characteristics.

We also observed that some strains within a given group were more resistant to storage conditions when compared with the others. This finding corroborates Voyron et al. [22] who reported that the application of a preservation protocol or one of its variables seldom produces the same result for all the isolates tested, even if they belong to the same genus or species. It seems that the response to different preservation techniques is strain specific, as observed by Ryan et al. [23] who found that the production of certain secondary metabolites by some strains of Metarhizium anisopliae was interrupted after cryopreservation and lyophilisation, whereas other strains of the same species were not affected.

The difficulty in recovering Malassezia spp. was also reported by Crespo et al. [1] who observed a low recovery rate after the application of different preservation methods. However, these authors obtained a high recovery rate, when the strains were stored at $-80^{\circ} \mathrm{C}$, after being lyophilised. The overall low recovery rate of Malassezia spp. from culture collections may be associated with the difficulty of obtaining homogeneous suspensions of lipodependent yeast. ${ }^{1}$ This fact was evidenced in the present study, where the viscosity of the immobilisation-cryopreservation medium caused an uneven distribution of the blastoconidia of Malassezia spp. that were imprisoned within the plastic beads. On the other hand, a uniform distribution of the zygomycete structures within the medium was successfully achieved.

Concerning the conservation of zygomycetes, some works report the cryopreservation of these fungal groups in culture collections. ${ }^{24-26}$ One work, written by Passarell \& McGinnis, ${ }^{24}$ reported the viability of several fungi, after storage at $-70{ }^{\circ} \mathrm{C}$, including some strains of zygomycetes. Similar to what was observed in the present study, a better recovery rate was obtained after storage at lower temperatures $\left(-70^{\circ} \mathrm{C} /-80^{\circ} \mathrm{C}\right)$. However, better results might have been obtained by storing these samples at ultralow temperatures (lower than $-139{ }^{\circ} \mathrm{C}$ ), as these temperatures avoid recrystallisation of ice and decrease the rate of biophysical processes that could affect cell survivability. ${ }^{27}$ Our results for the viability of zygomycetes are promising, considering the good recovery rate of the strains, especially within the first six months of storage.

In addition, these findings suggest that carbohydrates are effective cryoprotectant agents, as previously observed, ${ }^{16}$ most likely for stabilising cellular membranes and avoiding the formation of ice crystals, hence playing an important role in the protection of biological structures against the dehydration caused by cryopreservation methods. ${ }^{28}$ Finally, this study creates perspectives for the use of glucose and lactose associated with immobilisation of cells in sodium alginate for preserving zygomycetes, especially when kept at $-80{ }^{\circ} \mathrm{C}$, once it resulted in satisfactory recovery rates, after long period of storage.

\section{Acknowledgment}

We thank the research grants from the National Scientific and Technological Research Council; Brazil (CNPq, Protax 562296/2010-7 and Process 504189/2012-3).

\section{References}

1 Crespo MJ, Abarca ML, Cabañes FJ. Evaluation of different preservation and storage methods for Malassezia spp. J Clin Microbiol 2000; 38: 3872-5.

2 Guého E, Boekhout T, Ashbee HR, Guillot J, van Belkum A, Faergemann J. The role of Malassezia species in the ecology of human skin and as pathogens. Med Mycol 1998; 36: 220-9.

3 Lass-Florl C. Zygomycosis: conventional laboratory diagnosis. Clin Microbiol Infect 2009; 15: 60-5.

4 Nichols L, Ocque RZ, Daly I. Zygomycosis associated with HIV Infection and liver transplantation. Patholog Res Int 2011; 2011: 1-4.

5 Hernández-Lauzardo AN, Bautista-Baños S, Velásquez-del Valle MG, Trejo-Espino JL. Identification of Rhizopus stolonifer (Ehrenb.:Fr.) Vuill., Causal agent of Rhizopus Rot Disease of Fruis and Vegetables. Revista Mexicana de Fitopatología 2005; 24: 65-9.

6 Dworecka-Kaszak B, Toka FN. Comparison of different methods of maintenance Malassezia pachydermatis (s. Pityrosporum pachydermatis) strains. Acta Microbiol Pol 1996; 45: 103-5.

7 Breierová E, Kocková-Kratochvílová A, Delgado R. Storage of Candida albicans, Candida tropicalis and related species in liquid nitrogen. Folia Microbiol 1987; 32: 426-30.

8 Barbas JP, Mascarenha ERD. Cryopreservation of domestic animal sperm cells. Cell Tissue Bank 2009; 10: 49-62.

9 Benito MEG, Ramírez IC, Aranda LJM. The use of cryopreservation for germplasm conservation of vegetatively propagated crops. Span J Agric Res 2004; 2: 341-51.

10 Morgan CA, Heerman N, White PA, Vesey G. Preservation of microorganisms by drying. J Microbiol Methods 2006; 66: 183-93.

11 Benson EE. Cryopreservation of phytodiversity: a critical appraisal of theory and practice. CRC Crit Rev Plant Sci 2008; 27: 141-219.

12 Lewis JA, Papavizas GC. Characteristics of alginate pellets formulated with Trichoderma and Gliocladium and their effect on the proliferation of the fungi in soil. Plant Pathol 1985; 34: 571-7. 
13 Sag Y, Nourbakhsh M, Kutsal T. Comparison of Ca-alginate and immobilized Z. ramigera as sorbents for copper removal. Process Biochemical 1995; 30: 175-81.

14 Park C, Lee B, Hana E, Lee J, Kima S. Decolorization of acid black 52 by fungal immobilization. Enzyme Microb Technol 2006; 39: 371-4.

15 Prabakaran G, Hoti SL. Immobilization in alginate as a technique for the preservation of Bacillus thuringiensis var. israelensis for long-term. J Microbiol Methods 2008; 72: 91-4.

16 Soares-Júnior FA, Brilhante RSN, Cordeiro RA, Brito EHS, Sidrim JJC, Rocha MFG. Glucose improves the in vitro viability of Microsporum canis and Trichophyton mentagrophytes var. mentagrophytes. J Microbiol Methods 2007; 69: 218-21.

17 Hamoudi L, Goulet J, Ratti C. Effect of protective agents on the viability of Geotrichum candidum during freeze-drying and storage. J Food Sci 2007; 72: 45-9.

18 Ghého E, Midgley G, Guillot J. The genus Malassezia with description of four new species. Antonie Van Leeuwenhoek 1996; 69: 337-55.

19 Guillot J, Guého E, Lesourd M, Midgley G, Chévrier G, Dupont B. Identification of Malassezia species. A practical approach. J Mycol Med 1996; 6: 103-10.

20 Ribes JA, Vanover-Sams CL, Baker DJ. Zygomycetes in human disease. Clin Microbiol Rev 2000; 13: 236-301.
21 Espinel-Ingroff A, Montero D, Martin-Mazuelos E. Long-term preservation of fungal isolates in commercially prepared cryogenic microbank vials. J Clin Microbiol 2004; 42: 1257-9.

22 Voyron S, Roussel S, Munaut F et al. Vitality and genetic fidelity of white-rot fungi mycelia following different methods of preservation. Mycol Res 2009; 13: 1027-38.

23 Ryan JM, Jeffries P, Bridge PD, Smith D. Developing cryopreservation protocols secure fungal gene function. CryoLetters 2001; 22: 115-24.

24 Pasarell L, Mcginnis MR. Viability of fungal cultures maintained at $-70^{\circ} \mathrm{C}$. J Clin Microbiol 1991; 30: 1000-4.

25 Smith D, Onions AHS. The Preservation and Maintenance of Living Fungi, 2nd edn. IMI Technical Handbooks 2. Wallingford: CAB International, 1994: 1-120.

26 Homolka L, Lisá L, Kubátova A, Vánová M, Janderova B, Nerud F. Cryopreservation of filamentous micromicetes and yests using perlite. Folia Microbiol 2007; 52: 153-7.

27 Morris GJ. Cryopreservation. An Introduction to Cryopreservation in Culture Collections. Cambridge: Institute of Terrestrial Ecology, 1981.

28 Hincha DK, Rennecke P, Oliver AE. Protection of liposomes against fusion during drying by oligosaccharides is not predicted by the calorimetric glass transition temperatures of the dry sugars. Eur Biophys J 2008; 37: 503-8. 